\title{
A Study of the Functional Outcome of Galeazzi Fractures Treated with Open Reduction and Internal Fixation in Government Medical College Thrissur, Kerala
}

\author{
Abhilash Pillai ${ }^{1}$ Krishna Kumar C.V. ${ }^{2}$
}

${ }^{1}$ Department of Orthopaedics, Government Medical College, Thrissur, Kerala, India.

${ }^{2}$ Department of Orthopaedics, Government Medical College, Thrissur, Kerala, India.

\section{ABSTRACT}

\section{BACKGROUND}

In a Galeazzi fracture, a distal radius shaft fracture is accompanied by a disruption of the distal radio ulnar joint (DRUJ). $3 \%$ of all forearm fractures in children and 7 $\%$ of forearm fractures in adults are Galeazzi fractures. These may be due to direct or indirect trauma to the wrist. Mechanism of injury of a Galeazzi fracture is either due to a force applied onto the dorsolateral aspect of the forearm, or a fall on the outstretched hand with the forearm in pronation. In adults, Galeazzi fractures are treated by open reduction and compression plating of the radius and stabilization of the ulna if needed. We designed and carried out this study to find out the influence of the various factors associated with the fracture, its treatment and to find out which of these influenced the outcome.

\section{METHODS}

A prospective study was carried out in the Dept. of Orthopaedics, Government Medical College, Thrissur. All Galeazzi fractures presenting to the Orthopaedics Out Patient or Casualty during the study period were evaluated.

\section{RESULTS}

Post-operative rehabilitation is pivotal for ensuring an excellent functional outcome. Of all the parameters which were taken into account in the study, the only ones which made a difference was the period of immobilization in plaster cast given. Early mobilization yielded better functional result

\section{CONCLUSIONS}

25 cases Galeazzi fractures treated by open reduction done in our institution were followed up till union. The results were assessed with reference to the various parameters affecting fracture healing. Of all the factors which were considered, only the timing of removal of plaster and the physiotherapy given made any difference in the final functional result.

\section{KEY WORDS}

Galeazzi, Open Reduction, Forearm Fractures, Quick Dash Score, Functional Result.
Corresponding Author: Dr. Krishna Kumar C.V., Associate Professor, Department of Orthopaedics, Government Medical College, Trichur, Kerala, India.

E-mail: drkrishnakumarcv@gmail.com

DOI: $10.14260 / \mathrm{jemds} / 2021 / 788$

How to Cite This Article: Pillai A, Kumar KCV. A study of the functional outcome of Galeazzi fractures treated with open reduction and internal fixation in government medical college Thrissur, Kerala. J Evolution Med Dent Sci 2021;10(45):3900-3905, 10.14260/jemds/2021/788 DOI:

Submission 14-08-2021, Peer Review 22-08-2021, Acceptance 17-12-2021, Published 28-12-2021.

Copyright (C) 2021 Abhilash Pillai et al. This is an open access article distributed under Creative Commons Attribution License [Attribution 4.0 International (CC BY 4.0)] 


\section{BACKGROUND}

\section{Anatomy and Mechanism of Injury}

The radius is the main bone of the forearm, on the lateral side. Both proximal and distal are expanded for articulation, the distal end is broader than the proximal. The radius is much wider distally. It is convex laterally, and convex posteriorly. The radius articulates with ulna on both distally and proximally. Joints are formed by articulation with the carpus distally and the humerus proximally.

\section{Distal Radius}

The widest part of the distal radius is the distal end. It is four sided, and the lateral side has the projection called radial styloid. The medial side of the distal radial articular surface has a quadrangular shape. The lateral part of the distal radius is triangular and curves up to form the styloid process. The anterior surface of the distal radius is marked by a thick, palpable, prominent ridge, which is $2 \mathrm{~cm}$ proximal to the Thenar eminence. There is an anteroposterior concavity for articulation with the head of the radius on the medial surface, the ulnar notch.

\section{Distal Radio-ulnar Joint}

The distal radio-ulnar joint is a uniaxial pivot joint. It is formed by the convex distal head of the ulna which articulates with concave ulnar notch of the radius. There is an articular disc between them for connection

\section{Fibrous Capsule}

The fibrous capsule is thicker anteriorly and posteriorly, and more lax proximally.

Triangular fibrocartilage complex and distal radio-ulnar ligament is a ligamentous and cartilaginous structure that separates the distal radius and ulnar carpus from the distal ulna.

The triangular fibrocartilage complex (TFCC) stabilizes the ulnocarpal and radioulnar joints. It also transmits and distributes the load from the carpus to the ulna, thus facilitating complex movements at the wrist.

\section{Galeazzi Fracture \\ Classification}

There are several classification systems that have been used. According to Macule and colleagues, ${ }^{1}$ there are three types of lesion.

Type 1 lesion, the fracture of the radius occurs between 0 and $10 \mathrm{~cm}$ from the styloid process

Type 2 lesion, the fracture occurs between 10 and $15 \mathrm{~cm}$;

Type 3 lesion, the fracture occurs at more than $15 \mathrm{~cm}$ from the styloid process.

There is also a new treatment-oriented classification scheme suggested by Rettig and Raskin. ${ }^{2}$

Type 1 fracture- the distance between the mid articular surface of the distal radius and the fracture is within $7.5 \mathrm{~cm}$.

Type 2 fracture- the distance is more than $7.5 \mathrm{~cm}$.

In the type 1 fracture, the distal radioulnar joint was found to be significantly more unstable when it was tested intraoperatively after fixation of the radial fracture when compared with the type 2 fracture.

In the type 2 , only $6 \%$ of the patients required open fixation of the distal radioulnar joint.

The Galeazzi fracture-dislocation, according to Bruckner and colleagues, ${ }^{3}$ is also considered as simple or complex. When reduction is easy after fixation of the fracture, it is considered as simple. This is because in a complex dislocation, it is impossible to reduce or to keep the reduction because of interposition of soft tissue.

\section{Galeazzi Equivalents}

Certain injuries are considered as Galeazzi's equivalent lesions. A fracture of the radial shaft in children may be associated with separation of the distal ulnar epiphysis instead of a disruption of the DRUJ.

In adults, a fracture of the radial shaft may be associated with an additional fracture of the distal ulna instead of a disruption of the DRUJ.

These injuries are considered as Galeazzi's equivalent lesions and they are treated the same way as a Galeazzi fracture in children or in adults, respectively.4,5,6,7

\section{Mechanism of Injury}

The mechanism of injury is somewhat controversial. Though many authors believe that the injury occurs as a result of a fall on an outstretched and pronated hand, ${ }^{4}$ Other authors believe that the injury requires considerable force with an axial load and extremes of wrist extension and pronation.

Another cause has been reported to be a direct blow to the dorsoradial aspect of the forearm. ${ }^{8}$ Commonly obtained histories leading to a Galeazzi fracture include motor vehicle accidents, ${ }^{9}$ sports injuries, and falls from a height. An important soft-tissue stabilizer of the distal radioulnar joint is the triangular fibrocartilage complex. A rupture of the TFCC is thought to be the first step to dislocation and occurs at the extreme of pronation and extension of the wrist. $8,9,10,11,12,13,14,15,16$

\section{Natural History}

In an untreated fracture or one without a satisfactory reduction and stabilization, the pull from the different muscles that act on the fracture fragments causes a deformity of the distal forearm.

There is angulation and shortening of the radius with swelling, as well as tenderness and prominence of the ulnar head. ${ }^{8}$ In chronic instability and dislocation of the distal radioulnar joint, the wrist appears narrowed due to the pronator quadratus muscle, which approximates the ulna to the radius. 10,15

\section{Clinical Evaluation}

A patient who sustained a Galeazzi fracture-dislocation presents with swelling and deformity. Additional features are tenderness and pain to palpation of the distal forearm and wrist.

Usually, an angular deformity on the shortened radial side of the forearm can be seen. A protrusion of the ulnar head is also seen, which is found to be more mobile than usual. 
A high index of suspicion should be maintained by the examiner and a meticulous evaluation for instability of the distal radioulnar joint must be conducted.2,10,17 All of these signs and symptoms vary with the severity of injury and the degree of displacement. Subluxation or dislocation will be seen in the distal radioulnar joint, along with a prominence of the head of the ulna and also severe tenderness over the joint. It should be remembered that injury to the distal radioulnar joint occurs in approximately $20 \%$ of Galeazzi fractures, so any fracture of the distal third of the radius should be regarded as a potential Galeazzi fracture and the distal radioulnar joint should be examined very carefully. ${ }^{18,19,20}$

\section{Imaging}

A good radiographic evaluation is essential to have. This consists of anteroposterior (AP) and lateral radiographs of both the entire forearm and including the wrist. It is of great importance to identify a subluxation or a dislocation of the distal radioulnar joint.11,20 Radiographic appearance- the radius appears relatively shortened, and there is an increase in the space between the distal radius and ulna where they articulate.

In the lateral view, the fractured radius is usually angulated dorsally and the ulnar head is prominent dorsally. The injury to the radioulnar joint may be purely ligamentous or the ligament may remain intact and the ulnar styloid may be avulsed.10,19,21 Sometimes CT scanning may be helpful for the assessment of the dislocated distal radioulnar joint. ${ }^{22}$

There are four radiographic signs that suggest rupture of the distal radioulnar joint: (1) fracture at the base of the ulnar styloid, (2) widening of the DRUJ (AP view), (3) dislocation of the radius relative to the ulna (on a true lateral view), and (4) more than $5 \mathrm{~mm}$ of shortening of the radius relative to the ulna ("ulna plus," compared with the presumed normal ulnar variance in the opposite wrist). ${ }^{11,23}$

Meticulous evaluation of the TFCC can be performed by using MRI, arthrogram, arthroscopy, or a combination of these. 4

\section{Treatment / Non operative}

In children, although this type of injury is uncommon. ${ }^{4}$ Conservative management of Galeazzi fracture has been found to be successful. An excellent result ensues. As long as the fracture is immobilized in a long-arm cast in supination due to the thick periosteum. Open reduction sometimes is necessary; however, internal fixation is usually not required. ${ }^{10,19,24,25}$

In adults, conservative treatment results in a high percentage of failure. ${ }^{9,18}$ It has been observed that angulation and slipping of the radial fragments along with by subluxation or dislocation of the DRUJ can occur while the arm is in a cast, usually 7 to 10 days after the reduction.

This part is shortened and cut to half, not completely eliminated since some points are needed to show why surgical treatment is always justified and also some bibliography is quoted for the points.

\section{Surgical Management}

Campbell is said to have called this type of lesion "the fracture of necessity," by which he meant that open reduction and internal fixation was necessary if a good result were to be obtained. ${ }^{8}$

The radius fracture is approached through a volar "Henry" incision. Rigid internal fixation is imperative and provided by a $3.5-\mathrm{mm}$ or $4.5-\mathrm{mm}$ dynamic compression plate that should be of sufficient length and the screws must obtain good purchase in both cortices. ${ }^{10,19}$ After anatomic reduction and rigid fixation have been achieved, it is important to assess the adequacy and stability of reduction of the DRUJ. When marked instability of the joint is found, further treatment should be performed. Exploration of the joint with ligamentous repair or trans fixation of ulna and radius with K-wires after reposition of the dislocation is necessary.26,27 Presence or absence of an ulnar styloid fracture is also a deciding factor for need of further treatment.

In case, such a fracture is present, open reduction and internal fixation by using a screw or a K-wire with a tensionband technique will be required.9,10 These are the various options of treatment depending stability of the DRUJ are as follows.

\section{Stable DRUJ}

For healing of the volar and dorsal marginal ligaments of the joint, reduction of the DRUJ should be maintained with the forearm in supination for 6 weeks

\section{Unstable DRUJ}

Repair of the TFCC should be performed ${ }^{4}$ using sutures drilled through the ulna styloid or a suture anchor. Following repair, the DRUJ is pinned with a K-wire in neutral rotation to minimize loss of pronation. This repair has to be protected in plaster for 6 weeks.

\section{Unstable DRUJ with Ulna Styloid Fracture}

The ulna styloid fracture is usually through the fovea. The TFCC is attached to this fragment. Stability is obtained by open reduction and internal fixation of the styloid fragment, using a tension band wiring or a lag screw technique.

\section{Irreducible DRU J}

Soft tissue interposition may cause an inability to reduce the DRUJ. In these cases, open reduction can be accomplished and repair of the TFCC should be performed. Post operatively, a long-arm cast is generally used for 4 to 6 weeks according to the literature. $10,17,19,23,27$

\section{Postoperative Care}

Even after a rigid fixation has been performed, the patient should be placed in a long-arm cast for 4 to 6 weeks. This is to allow sufficient healing of the soft tissues.

Suture removal- this is after approximately 2 weeks. After cast removal, active and passive exercises of the elbow, wrist, and forearm are begun. Emphasis is given, on regaining pronation and supination. The wrist can be protected in a removable splint for an additional 3 to 4 weeks. ${ }^{10,17,19,27}$ 


\section{Complications}

The complications of Galeazzi fracture-dislocations are the same as those of all forearm fractures. These are-non-union, delayed union, malunion, nerve injuries, and infection. other complications like reflex sympathetic dystrophy, synovitis, reduced motion, and refractures after plate removal may also be seen. The most common complication is angulation at the fracture site and subluxation or dislocation of the DRUJ.28

\section{Aims \& Objectives}

To assess functional outcome of Galeazzi fracture treated with open reduction and internal fixation in patients attending Orthopaedics OPD in Government Medical College Thrissur.

\section{METHODS}

A prospective study was conducted in the Dept. of Orthopaedics, Government Medical College, Thrissur. Study period was for 18 months from $01-01-2017$ to $30-06$ 2018. All adults with Galeazzi fractures attending the Orthopaedics Department were included in the study.

\section{Sample Size}

25: All cases both new and old (coming for follow up) to the orthopaedics OPD or casualty during the study period were considered since the patients with Galeazzi fracture treated with ORIF is low.

$\mathrm{n}=(\mathrm{Z} \alpha)^{2} \times \mathrm{p} \times \mathrm{q} / \mathrm{d}^{2}$

$\mathrm{n}=$ sample size

$\mathrm{p}=$ proportion of patients with Galeazzi fracture having a good outcome with ORIF in study named "Functional Outcome of Galeazzi Fractures Treated by ORIF and DRUJ Stabilization either Using Long Arm Cast or Transfixing Wire" done by Dewo P, Yudhistira JF, Lanodiyu Z and Magetsari R, Department of Orthopaedics and Traumatology, Yogyakarta, Indonesia. ${ }^{29}$

$\mathrm{q}=100-\mathrm{p}$

$\mathrm{d}=$ maximum allowable error $(20 \%$ of $\mathrm{p})$

$\mathrm{Z} \alpha=$ constant $=1.96$

$\mathrm{n}=(1.96)^{2} \times 79 \times 21 /(0.2 \times 79)^{2}$

$=25$

\section{Sampling Method}

Convenient sampling

\section{Statistical Analysis}

Data entered into MS Excel and was analysed. Qualitative variables were analysed using proportions.

\section{Inclusion Criteria}

Patients diagnosed with Galeazzi fracture attending OPD Orthopaedics (both type 1 and 2).

\section{Exclusion Criteria}

1. Patients with significant disabling co morbidities like cancer and neurodegenerative disorders.

2. Patients with mental retardation.

3. Patients less than 18 years of age

4. Patients not giving consent

\section{Ethical considerations}

The study commenced after obtaining clearance from Institutional Ethics Committee and Institutional Review Board and written informed consent from patients.

\section{Scoring System Used}

The scoring system used was quick DASH score. It is an abbreviated version of the original DASH score. This is a 11item questionnaire which assesses activity, function and symptoms like daily activities, house / yard work, shopping, recreation, self-care, eating, sleep, work, pain, tingling / numbness. It also contains two optional modules on sport / music or work.

The greater the score, more the disability. It is available at www.dash.iwh.on.ca

\section{Data Collection}

For this study patients were selected based on inclusion and exclusion criteria. The following data was obtained for all study patients as per the proforma attached below. Age, sex, nature of trauma, side of fracture, any associated injuries and comorbidities. Any pain, swelling, and loss of functions noted. Informed consent was obtained from all patients prior to surgery. Anteroposterior and lateral view X-rays of involved forearm with elbow and wrist were taken. Routine blood investigations were performed. Detailed history was taken with particular enquiry on mode of injury. Adequate treatment was given for other associated medical problems. Patient and attenders were informed about surgery and risk factors. Pre-operative anaesthetic evaluations for anaesthetic fitness were carried out. Intravenous antibiotic was given an hour before surgery.

\section{Operative Procedure}

ORIF WITH 3.5 DCP was the method of choice. DRUJ fixation was done only in cases of clinical instability under General anaesthesia / Block, Radius is approached via Volar Approach of Henry by exposure of the radius from the bicipital tuberosity to the distal articular surface of the radius can be achieved using the anterior approach 72 .

Fracture was reduced and fixed with $3.5 \mathrm{~mm}$ DCP. Reduction assessed. DRUJ instability examined. In our series, we had no cases of obvious clinical instability which required DRUJ K wire fixation. After giving thorough wash, wound closed in layers. Long arm slab was applied.

\section{Post-Operative Care}

Limb is maintained in supination and long arm slab. Postoperative rehabilitation was started at 4 to 6 weeks after removing the cast. 


\section{Scoring System}

The scoring system used is the quick DASH score

\section{RESULTS}

25 cases of Galeazzi fractures treated with open reduction internal fixation were studied and analysed and the following results were obtained. Out of 25 patients, 20 (80\%) were males and $5(20 \%)$ were females with age distribution ranging from 19 - 64 years and maximum number of patients falling in the $31-40$ years age group. Of them, $13(52 \%)$ had right Galeazzi fracture while 12 (48 \%) had left sided fracture. The mechanism of injury in $20(80 \%)$ was fall, 4 (16 $\%)$ were RTA and 1 (4\%) from other causes. Of this, 18 (72 $\%)$ was of type 1 fracture and 7 (28 \%) was of type 2 fracture. Regarding the comorbidities, 4 (16 \%) had medical comorbidities, 3 (12\%) had high energy trauma, 1 (4\%) having open fracture. Surgery was done within 12 hours in 7 (28\%), 12 - 24 hours in 10 (40\%), 24-48 hours in 7 (28\%) and after 48 hours in 1 (4\%). Regarding surgery duration, 15 (60\%) cases were done within 45 minutes and 10 (40\%) were done in 45 - 60 minutes. 15 (60\%) cases were discharged within 3 days, 6 (24\%) were discharged between 4 - 7 days, 2 (8\%) were discharged between 8 - 10 days and 2 (8\%) were discharged between 11 - 14 days.

Follow up, investigations, and data collection: all cases were followed up with regular $\mathrm{x}$ rays, clinical assessment using quick DASH score, and hemogram in case of suspicion of infection if needed. This was done at intervals of 30 days till complete fracture union and maximum functional result.

\section{Follow up in Detail and Methods Used}

2 (8\%) patients developed infection and no early complication in other cases. No other early complications manifested in our study. Late post-operative complications included DRUJ instability in 3 (12\%), delayed union in 1 (4 $\%)$ and malunion in 1 (4\%). According to Quick Dash Score, $20(80 \%)$ had good functional outcome, 4 (16\%) had fair functional outcome and 1 (4\%) had poor outcome. None of the patients had severe disability.

\section{Treatment of Complications}

Infections were treated with antibiotics and short period of immobilization. No surgical interventions were needed, and all cases settled with no disability. Other complications were not severe enough to warrant intervention since disability was minimal.

\section{DISCUSSION}

\section{Sex Distribution}

In our study among the 25 cases, 20 (80\%) were males and $5(20 \%)$ were females. Our study is comparable with the preceding studies. This is probably due to the activity level and the propensity of men to perform more outdoor activities.

\section{Age Distribution}

In our study age of patients ranged from 19 - 64 years and maximum number of patients fell in the $31-40$ years age group. It is to be surmised that incidence of this fracture is higher in active/employed group.

\section{Type of Galeazzi Fracture}

Out of the 25 patients in our study, 18 (72\%) were of type 1 and 7 (28\%) were of type 2 Galeazzi fracture. Other types were not seen. Type 1 forms the absolute majority of the fracture types seen

\section{Functional outcome}

In our study among 25 patients, 20 (80\%) had good functional outcome, 4 (16\%) had fair functional outcome \& 1 (4\%) had poor outcome. None of the patients had severe disability as functional outcome. Though this was a study done under controlled settings in a tertiary referral hospital, it is obvious that majority of the patients who sustained this injury have a good outcome, and permanent disability is rare.

\section{Summary}

We have done a prospective study for finding out the functional outcome of Galeazzi fracture treated with open reduction internal fixation in 25 cases over a period of 18 months in the Department of Orthopaedics Govt Medical College Thrissur.

In our study among 25 patients, $80 \%$ were males and $20 \%$ were females with patient's ranging from $19-64$ years with maximum number of patients falling in the 31 40 years age group. Side of injury: $52 \%$ subjects with right Galeazzi fracture and $48 \%$ with left sided fracture. Mechanism of injury: $80 \%$ were due to fall. Type of fracture: $72 \%$ sustained a type 1 fracture. All patients were treated with an open reduction internal fixation within 48 hours of injury in $96 \%$ cases and $92 \%$ discharged from hospital within 10 days. $8 \%$ developed infection in early post-operative period and $12 \%$ of patients developed DRUJ instability on an average follow up of 6 months. $80 \%$ had good functional outcome, $16 \%$ had fair functional outcome and $1 \%$ had poor outcome.

\section{CONCLUSIONS}

The conclusion that can be drawn from the above study is that in the event of Galeazzi fracture the most befitting treatment is anatomic restoration of length of the radius with application of rigid internal fixation to maintain the reduction. Only 1 patient out of the 25 cases had poor result with timely ORIF and post-operative mobilization. A high index of suspicion should be maintained by the surgeon, and a thorough examination for instability of the DRUJ must be conducted. In 3 cases of DRUJ instability recorded in this study, patients were asymptomatic, and a mild instability was noted only on post-operative assessment. Postoperative rehabilitation is pivotal for ensuring an excellent functional outcome. 
Of all the parameters which were taken into account in the study, the only one which made a difference was the period of immobilization of plaster cast given.

Early mobilization yielded better functional results. We recommend that all cases of Galeazzi fracture be given the minimum possible period of immobilization in plaster cast following ORIF for best functional results.

Data sharing statement provided by the authors is available with the full text of this article at jemds.com.

Financial or other competing interests: None.

Disclosure forms provided by the authors are available with the full text of this article at jemds.com.

\section{REFERENCES}

[1] Beneyto FM, Renu JMR, Claramunt AF, et al Treatment of Galeazzi fracture-dislocations. J Trauma 1994;36(3):3525.

[2] Rettig ME, Raskin KB. Galeazzi fracture-dislocation: a new treatment-oriented classification. J Hand Surg Am 2001;26(2):228-35.

[3] Bruckner JD, Lichtman DM, Alexander AH. Complex dislocations of the distal radioulnar joint. Recognition and management. Clin Orthop Relat Res 1992;(275):90103.

[4] Mikić ZD Galeazzi fracture-dislocations. J Bone Joint Surg Am 1975;57(8):1071-80.

[5] Reckling FW. Unstable fracture-dislocations of the forearm (Monteggia and Galeazzi lesions). J Bone Joint Surg Am 1982;64(6):857-63.

[6] Imatani J, Hashizume H, Nishida K, et al. The Galeazziequivalent lesion in children revisited. J Hand Surg Br 1996;21(4):455-7.

[7] Kamano M, Honda Y. Galeazzi-equivalent lesions in adolescence. J Orthop Trauma 2002;16(6):440-3.

[8] Aulicino PL, Siegel JL. Acute injuries of the distal radioulnar joint. Hand Clin 1991;7(2):283-93.

[9] Hughston JC. Fracture of the distal radial shaft; mistakes in management. J Bone Joint Surg Am 1957;39-A(2):24964.

[10] Haugstvedt JR. Dissociations of the radius and ulna: surgical anatomy and biomechanics. In: Berger RA, Weiss APC, eds. Hand surgery. Philadelphia: Lippincott Williams \& Wilkins 2004:615-44.

[11] Schneiderman G, Meldrum RD, Bloebaum RD, et al. The interosseous membrane of the forearm: structure and its role in Galeazzi fractures. J Trauma 1993;35(6):879-85.

[12] Wallace $A L$, Walsh WR, van Rooijen $M$, et al. The interosseous membrane in radio-ulnar dissociation. J Bone Joint Surg Br 1997;79(3):422-7.
[13] Almquist EE. Evolution of the distal radioulnar joint. Clin Orthop Relat Res 1992;275:513.

[14] Linscheid RL. Biomechanics of the distal radioulnar joint. Clin Orthop Relat Res 1992;275:46-55.

[15] Palmer AK, Werner FW. Biomechanics of the distal radioulnar joint. Clin Orthop Relat Res 1984;187:26-35.

[16] Palmer AK, Werner FW. The triangular fibrocartilage complex of the wrist anatomy and function. J Hand Surg Am 1981;6(2):153-62.

[17] Bruckner JD, Lichtman DM, Alexander AH. Complex dislocations of the distal radioulnar joint. Recognition and management. Clin Orthop Relat Res 1992;(275):90103.

[18] Ring D, Rhim R, Carpenter C, et al. Isolated radial shaft fractures are more common than Galeazzi fractures. J Hand Surg Am 2006;31(1):17-21.

[19] Renfree KJ. Shaft fractures of the radius and ulna. In: Berger RA, Weiss APC, eds. Hand surgery. Philadelphia: Lippincott Williams \& Wilkins 2004:577-614.

[20] Nicolaidis SC, Hildreth DH, Lichtman DM. Acute injuries of the distal radioulnar joint. Hand Clin 2000;16(3):44959.

[21] Burk DL, Karasick D, Wechler RJ. Imaging of the distal radioulnar joint. Hand Clin 1991;7(2):263-75.

[22] Mino DE, Palmer AK, Levinsohn EM. The role of radiography and computerized tomography in the diagnosis of subluxation and dislocation of the distal radioulnar joint. J Hand Surg Am 1983;8(1):23-31.

[23] Moore TM, Klein JP, Patzakis JP, et al. Results of compression-plating of closed Galeazzi fractures. J Bone Joint Surg Am 1985;67(7):1015-21.

[24] Ooi LH, Toh CL. Galeazzi-equivalent fracture in children associated with tendon entrapment report of two cases. Ann Acad Med Singapore 2001;30(1):51-4.

[25] Cetti NE. An unusual cause of blocked reduction of the Galeazzi injury. Injury 1977;9(1):59-61.

[26] Strehle J, Gerber C. Distal radioulnar joint function after Galeazzi fracture-dislocations treated by open reduction and internal plate fixation. Clin Orthop Relat Res 1993;(293):240-5.

[27] Adams BD, Berger RA. An anatomic reconstruction of the distal radioulnar ligaments for posttraumatic distal radioulnar joint instability. J Hand Surg Am 2002;27(2):243-51.

[28] Gosselin RA, Contreras DM, Delgado E, et al. Anterior dislocation of the distal end of the ulna after use of a compression plate for the treatment of a Galeazzi fracture. A case report. J Bone Joint Surg Am 1993;75(4):593-6.

[29] Dewo P, Yudhistira JF, Lanodiyu Z, et al. Functional outcome of galeazzi fractures treated by ORIF and DRUJ stabilization either using long arm cast or transfixing wire. Orthop Muscular Syst 2015;4(3):192. 